\title{
ON EQUATIONAL DEFINABILITY OF FUNCTION CLASSES
}

\author{
MIGUEL COUCEIRO, ERKKO LEHTONEN, AND TAMÁS WALDHAUSER
}

\begin{abstract}
We propose a notion of functional equation for functions of a fixed arity, which is based on a pair of clones. We present necessary conditions for a class of functions to be definable by such equations, and show that for certain choices of clones these conditions are also sufficient.
\end{abstract}

\section{INTRODUCTION AND MOTIVATIONS}

This paper is a study of definability of properties of functions by functional equations. We propose an equational framework which differs from those presented in $[5,7,12,13,14,23$, in that here the properties to be defined concern functions of a given fixed arity rather than functions of different arities. (This distinction is made clear in Subsection 1.2.) The current approach is thus rooted in the classical theory of functional equations, and it provides a means to express natural properties of functions that are not definable in those other equational frameworks. A classical example of such a property is symmetry. A preliminary version of the current paper was presented at the 41st International Symposium on Multiple-Valued Logic (ISMVL 2011), see 9].

1.1. Basic notions. Throughout the paper let $A, B$ and $C$ be finite sets, and for each integer $n \geq 1$ let $[n]:=\{1, \ldots, n\}$. We denote tuples by bold letters and their components by corresponding indexed italic letters, e.g., $\mathbf{a}=\left(a_{1}, \ldots, a_{n}\right) \in A^{n}$. By a function of several variables from $A$ to $B$ (or simply function, when the sets $A$ and $B$ are clear from the context) we mean a map $f: A^{n} \rightarrow B$, where $n \geq 0$ is called the arity of $f$. The set of all $n$-ary functions from $A$ to $B$ is denoted by $B^{A^{n}}$. For a class $\mathcal{K} \subseteq \bigcup_{n \geq 0} B^{A^{n}}$, we set $\mathcal{K}^{(m)}=\mathcal{K} \cap B^{A^{m}}$. Functions of several variables from the two-element set $\{0,1\}$ to $\{0,1\}$ are usually called Boolean functions. The set of all functions of several variables from $A$ to $A$ is denoted by $\mathcal{O}_{A}$. The kernel of a function $f \in B^{A^{n}}$ is the equivalence relation ker $f \subseteq A^{n} \times A^{n}$ defined by $\left(\mathbf{a}, \mathbf{a}^{\prime}\right) \in \operatorname{ker} f \Longleftrightarrow f(\mathbf{a})=f\left(\mathbf{a}^{\prime}\right)$.

The composition of $f: B^{n} \rightarrow C$ by $g_{1}, \ldots, g_{n}: A^{m} \rightarrow B$, denoted by $f\left(g_{1}, \ldots, g_{n}\right)$, is defined as the $m$-ary function from $A$ to $C$ given by

$$
f\left(g_{1}, \ldots, g_{n}\right)(\mathbf{a})=f\left(g_{1}(\mathbf{a}), \ldots, g_{n}(\mathbf{a})\right), \text { for every } \mathbf{a} \in A^{m} .
$$

We say that $f$ is the outer function of the composition, and $g_{1}, \ldots, g_{n}$ are the inner functions.

A clone on $A$ is a class $\mathcal{C} \subseteq \mathcal{O}_{A}$ of finitary functions on $A$ that is closed under composition and contains the projections

$$
x_{i}^{(n)}: A^{n} \rightarrow A, \quad\left(x_{1}, \ldots, x_{n}\right) \mapsto x_{i} \quad(n \in \mathbb{N}, 1 \leq i \leq n) .
$$

We will omit the upper index, when there is no risk of ambiguity. It is noteworthy that the class $\mathcal{I}_{A}$ of all projections is the smallest clone on $A$, whereas $\mathcal{O}_{A}$ is the largest clone on $A$. Also, it is well known that the intersection of any family of clones is itself a clone. Hence, for $F \subseteq \mathcal{O}_{A}$, there is a smallest clone on $A$ which contains $F$, namely, $\langle F\rangle_{A}:=\bigcap_{C \in \mathcal{F}} C$, where $\mathcal{F}$ is the set of all clones on $A$ which contain $F$; we may write simply $\langle F\rangle$ whenever the underlying set $A$ is clear from the context. We will denote the clone of all constant functions and projections on $A$ by $\mathcal{C}_{A}$. For further background in clone theory see, e.g., [19, 24]. 
1.2. Functional equations. In [7, the authors worked on an equational framework for defining properties of functions $f: A^{n} \rightarrow B, n \geq 1$, rooted in universal algebra and originally proposed in 12 for the study of Boolean functions. Essentially, a functional equation (for functions $f: A^{n} \rightarrow B$ ) was defined as a formal expression

$$
\begin{aligned}
u\left(\mathbf{f}\left(g_{1}\left(\mathbf{x}_{1}, \ldots, \mathbf{x}_{m}\right)\right), \ldots, \mathbf{f}\left(g_{r}\left(\mathbf{x}_{1}, \ldots, \mathbf{x}_{m}\right)\right)\right) & \\
& =v\left(\mathbf{f}\left(h_{1}\left(\mathbf{x}_{1}, \ldots, \mathbf{x}_{m}\right)\right), \ldots, \mathbf{f}\left(h_{s}\left(\mathbf{x}_{1}, \ldots, \mathbf{x}_{m}\right)\right)\right),
\end{aligned}
$$

where $r, s, m \geq 1, u: B^{r} \rightarrow C, v: B^{s} \rightarrow C$, each $g_{i}$ and $h_{j}$ is a map $A^{m} \rightarrow A$, the symbols $\mathbf{x}_{1}, \ldots, \mathbf{x}_{m}$ are $m$ distinct vector variable symbols, and $\mathbf{f}$ is a distinct function symbol. A function $f: A^{n} \rightarrow B$ is said to satisfy (1) if, for all $\mathbf{a}_{1}, \ldots, \mathbf{a}_{m} \in$ $A^{n}$,

$$
\begin{aligned}
u\left(f\left(g_{1}\left(\mathbf{a}_{1}, \ldots, \mathbf{a}_{m}\right)\right), \ldots, f\left(g_{r}\left(\mathbf{a}_{1}, \ldots, \mathbf{a}_{m}\right)\right)\right) & \\
& =v\left(f\left(h_{1}\left(\mathbf{a}_{1}, \ldots, \mathbf{a}_{m}\right)\right), \ldots, f\left(h_{s}\left(\mathbf{a}_{1}, \ldots, \mathbf{a}_{m}\right)\right)\right) .
\end{aligned}
$$

In this way, a class of functions is defined by a set of such functional equations if it comprises exactly those functions which satisfy every equation in the set.

This framework has been advantageously used to specify noteworthy properties of functions in terms of functional equations. Classical examples include

- linearity of functions $f: \mathbb{F}^{n} \rightarrow \mathbb{F}, n \geq 1$, on a field $\mathbb{F}$, which is defined by

$$
\mathbf{f}(\mathbf{x}+\mathbf{y})=\mathbf{f}(\mathbf{x})+\mathbf{f}(\mathbf{y})
$$

- nondecreasing and nonincreasing monotonicity of functions $f: L^{n} \rightarrow L$, $n \geq 1$, on a lattice $L$, which are defined by

$$
\mathbf{f}(\mathbf{x})=\mathbf{f}(\mathbf{x}) \vee \mathbf{f}(\mathbf{x} \wedge \mathbf{y}) \quad \text { and } \quad \mathbf{f}(\mathbf{x})=\mathbf{f}(\mathbf{x}) \vee \mathbf{f}(\mathbf{x} \vee \mathbf{y})
$$

respectively.

More contemporary examples, which have strong consequences in combinatorial optimization, include

- submodularity of functions $f: I^{n} \rightarrow \mathbb{R}$, where $I$ is a chain, usually expressed by the functional inequality

$$
\mathbf{f}(\mathbf{x})+\mathbf{f}(\mathbf{y}) \geq \mathbf{f}(\mathbf{x} \wedge \mathbf{y})+\mathbf{f}(\mathbf{x} \vee \mathbf{y})
$$

or, equivalently, by the functional equation

$$
\mathbf{f}(\mathbf{x})+\mathbf{f}(\mathbf{y})=\max (\mathbf{f}(\mathbf{x} \wedge \mathbf{y})+\mathbf{f}(\mathbf{x} \vee \mathbf{y}), \mathbf{f}(\mathbf{x})+\mathbf{f}(\mathbf{y}))
$$

- supermodularity of functions $f: I^{n} \rightarrow \mathbb{R}$, where $I$ is a chain, usually expressed by the functional inequality

$$
\mathbf{f}(\mathbf{x})+\mathbf{f}(\mathbf{y}) \leq \mathbf{f}(\mathbf{x} \wedge \mathbf{y})+\mathbf{f}(\mathbf{x} \vee \mathbf{y})
$$

or, equivalently, by the functional equation

$$
\mathbf{f}(\mathbf{x})+\mathbf{f}(\mathbf{y})=\min (\mathbf{f}(\mathbf{x} \wedge \mathbf{y})+\mathbf{f}(\mathbf{x} \vee \mathbf{y}), \mathbf{f}(\mathbf{x})+\mathbf{f}(\mathbf{y}))
$$

- modularity of functions $f: I^{n} \rightarrow \mathbb{R}$, where $I$ is a chain, which is defined by the functional equation

$$
\mathbf{f}(\mathbf{x})+\mathbf{f}(\mathbf{y})=\mathbf{f}(\mathbf{x} \wedge \mathbf{y})+\mathbf{f}(\mathbf{x} \vee \mathbf{y})
$$

Using this functional equation, it can be shown [26] that modular functions are exactly those which are separable, i.e., of the form $\sum f_{i}\left(x_{i}\right)+c$, where $f_{i}: I \rightarrow \mathbb{R}$ for each $i \in[n]$ and $c \in \mathbb{R}$. For background on sub- and supermodularity and their applications in combinatorial optimization, see, e.g., [20, 27.

Pippenger 23. considered somewhat different defining objects, so-called relational constraints. An $m$-ary relational constraint from $A$ to $B$ is a pair $(R, S)$, 
where $R$ is an $m$-ary relation on $A$ and $S$ is an $m$-ary relation on $B$. A function $f: A^{n} \rightarrow B$ preserves a relational constraint $(R, S)$, if for all $m$-by- $n$ matrices $\mathbf{A}=\left[a_{i j}\right]_{m \times n}$ with entries from $A$, it holds that

$$
\left(f\left(a_{11}, \ldots, a_{1 n}\right), f\left(a_{21}, \ldots, a_{2 n}\right), \ldots, f\left(a_{m 1}, \ldots, a_{m n}\right)\right) \in S
$$

whenever $\left(a_{1 j}, \ldots, a_{m j}\right) \in R$ for all $j \in[n]$ (in other words, if all columns of $\mathbf{A}$ are tuples in the relation $R$, then application of $f$ to the rows of $\mathbf{A}$ results in a tuple in $S$ ). A class $\mathcal{C}$ of functions is defined by a set $\mathcal{K}$ of relational constraints if $\mathcal{C}$ is the set of all functions that preserve all relational constraints in $\mathcal{K}$.

As it turned out, relational constraints have the same expressive power as functional equations in the sense that they define exactly the same function classes. For example, let $I$ be a chain and let

$$
\begin{aligned}
R & =\left\{(a, b, c, d) \in I^{4}: c=a \wedge b, d=a \vee b\right\}, \\
S_{\geq} & =\left\{(p, q, r, s) \in \mathbb{R}^{4}: p+q \geq r+s\right\}, \\
S_{\leq} & =\left\{(p, q, r, s) \in \mathbb{R}^{4}: p+q \leq r+s\right\}, \\
S_{=} & =\left\{(p, q, r, s) \in \mathbb{R}^{4}: p+q=r+s\right\} .
\end{aligned}
$$

It is not difficult to see that $\left(R, S_{\geq}\right),\left(R, S_{\leq}\right)$, and $\left(R, S_{=}\right)$define the classes of submodular, supermodular, and modular functions, respectively.

Observe that a function $f: A^{n} \rightarrow A$ preserves a relation $R$ if and only if $f$ preserves the relational constraint $(R, R)$. Since clones (on a finite set) are exactly those classes which are defined by relations [3, 15, 19, 24, every clone is definable by relational constraints or, equivalently, by functional equations. For variants and extensions see, e.g., [5, 8, 13, 14, 22, 23.

Classes definable by functional equations of the form (1), or, equivalently, by relational constraints, were completely characterized in terms of a quasi-ordering of functions, the so-called simple minor relation. For a finite set $A$, the equational classes of $B$-valued functions on $A$ were shown to coincide with the initial segments of this quasi-ordering [4, 11, 12, 23. In the case when $A$ is arbitrary, possibly infinite, one additional "local closure" condition is also required [6, 7].

Despite the fact that a wide variety of function classes can be defined within this framework, it cannot express certain classical properties of functions $f: A^{n} \rightarrow B$ such as symmetry:

$$
f\left(a_{1}, \ldots, a_{n}\right)=f\left(a_{\sigma(1)}, \ldots, a_{\sigma(n)}\right)
$$

for all $a_{1}, \ldots, a_{n} \in A$ and any permutation $\sigma$ on $[n]$. This limitation may be due to the fact that functional equations of the form (1) do not refer to the arity of functions. This fact leads to the following notion of functional equation, that is rooted in classical theory of functional equations.

Let $\mathcal{A}$ and $\mathcal{B}$ be clones on $A$ and $B$, respectively. $\mathrm{A}(\mathcal{B}, \mathcal{A})$-equation is a functional equation of the form

$$
\begin{aligned}
u\left(\mathbf{f}\left(g_{11}, \ldots, g_{1 n}\right), \ldots, \mathbf{f}\left(g_{r 1}, \ldots,\right.\right. & \left.\left.g_{r n}\right)\right) \\
& =v\left(\mathbf{f}\left(h_{11}, \ldots, h_{1 n}\right), \ldots, \mathbf{f}\left(h_{s 1}, \ldots, h_{s n}\right)\right),
\end{aligned}
$$

where $r, s, n \geq 0, u \in \mathcal{B}^{(r)}, v \in \mathcal{B}^{(s)}$, each $g_{i j}$ and $h_{i j}$ is a function in $\mathcal{A}^{(m)}, m \geq 0$, and $\mathbf{f}$ is an $n$-ary function symbol. Observe that if we interpret the function symbol f by a function $f: A^{n} \rightarrow B$, then each side of (2) becomes an $m$-ary function from $A$ to $B$. For this reason we will sometimes refer to $(2)$ as an $m$-ary $(\mathcal{B}, \mathcal{A})$-equation.

We say that $f: A^{n} \rightarrow B$ satisfies $(2)$ if for every $\mathbf{a} \in A^{m}$

$$
\begin{aligned}
u\left(f\left(g_{11}, \ldots, g_{1 n}\right), \ldots, f\left(g_{r 1}, \ldots, g_{r n}\right)\right)(\mathbf{a}) & \\
& =v\left(f\left(h_{11}, \ldots, h_{1 n}\right), \ldots, f\left(h_{s 1}, \ldots, h_{s n}\right)\right)(\mathbf{a}) .
\end{aligned}
$$

A set $\mathcal{E}$ of $(\mathcal{B}, \mathcal{A})$-equations defines a class $\mathcal{K}$ of $n$-ary functions from $A$ to $B$ if $f \in \mathcal{K}$ if and only if $f$ satisfies all members of $\mathcal{E}$. A class $\mathcal{K}$ of $n$-ary functions from $A$ to $B$ is (finitely) definable by $(\mathcal{B}, \mathcal{A})$-equations if there is a (finite) set $\mathcal{E}$ of 
$(\mathcal{B}, \mathcal{A})$-equations that defines $\mathcal{K}$. In the sequel, unless otherwise specified, $\mathcal{B}$ and $\mathcal{A}$ always denote arbitrary clones on $B$ and $A$.

Example 1. The class of $n$-ary symmetric functions is definable by $\left(\mathcal{I}_{A}, \mathcal{I}_{A}\right)$ equations. Indeed, $f \in \mathcal{O}_{A}^{(n)}$ is symmetric if and only if it satisfies the $\left(\mathcal{I}_{A}, \mathcal{I}_{A}\right)$ equation

$$
f\left(x_{1}, \ldots, x_{n}\right)=f\left(x_{\sigma(1)}, \ldots, x_{\sigma(n)}\right),
$$

for every permutation $\sigma$ on $[n]$. In fact, two of these $n$ ! equations suffice, since the symmetric group $S_{n}$ can be generated by two permutations (e.g., an $n$-cycle and a transposition).

Example 2. Let $\left(A ; \wedge_{A}, \vee_{A}\right)$ and $\left(B ; \wedge_{B}, \vee_{B}\right)$ be lattices. The class of $n$-ary orderpreserving functions from $A$ to $B$ is defined by the $\left(\left\langle\wedge_{B}\right\rangle,\left\langle\wedge_{A}\right\rangle\right)$-equation

$$
\mathbf{f}\left(x_{1} \wedge_{A} y_{1}, \ldots, x_{n} \wedge_{A} y_{n}\right) \wedge_{B} \mathbf{f}\left(x_{1}, \ldots, x_{n}\right)=\mathbf{f}\left(x_{1} \wedge_{A} y_{1}, \ldots, x_{n} \wedge_{A} y_{n}\right),
$$

while the class of $n$-ary order-reversing functions from $A$ to $B$ is defined by the $\left(\left\langle\wedge_{B}\right\rangle,\left\langle\wedge_{A}\right\rangle\right)$-equation

$$
\mathbf{f}\left(x_{1} \wedge_{A} y_{1}, \ldots, x_{n} \wedge_{A} y_{n}\right) \wedge_{B} \mathbf{f}\left(x_{1}, \ldots, x_{n}\right)=\mathbf{f}\left(x_{1}, \ldots, x_{n}\right) .
$$

Similarly, these classes are definable by $(\mathcal{B}, \mathcal{A})$-equations for any choice of clones $\mathcal{B} \in$ $\left\{\left\langle\wedge_{B}\right\rangle,\left\langle\vee_{B}\right\rangle\right\}$ and $\mathcal{A} \in\left\{\left\langle\wedge_{A}\right\rangle,\left\langle\vee_{A}\right\rangle\right\}$. Clearly, all of these equations are $\left(\mathcal{M}_{B}, \mathcal{M}_{A}\right)$ equations, where $\mathcal{M}_{A}$ and $\mathcal{M}_{B}$ denote the clones of monotone (order-preserving) functions on $A$ and $B$, respectively. In general, the larger the clones $\mathcal{B}$ and $\mathcal{A}$ are, the larger the expressive power of $\left(\mathcal{B}, \mathcal{A}\right.$ )-equations is: if $\mathcal{B} \subseteq \mathcal{B}^{\prime}$ and $\mathcal{A} \subseteq \mathcal{A}^{\prime}$, then every class definable by $(\mathcal{B}, \mathcal{A})$-equations is definable by $\left(\mathcal{B}^{\prime}, \mathcal{A}^{\prime}\right)$-equations.

Example 3. If $(A ; \cdot)$ and $(B ; *)$ are groupoids, with clones of term functions $\mathcal{A}$ and $\mathcal{B}$, respectively, then the set of homomorphisms from $A^{n}$ to $B$ is defined by the $(\mathcal{B}, \mathcal{A})$-equation

$$
\mathbf{f}\left(x_{1} \cdot y_{1}, \ldots, x_{n} \cdot y_{n}\right)=\mathbf{f}\left(x_{1}, \ldots, x_{n}\right) * \mathbf{f}\left(y_{1}, \ldots, y_{n}\right) .
$$

This is a generalization of the classical Cauchy equation $f(x+y)=f(x)+f(y)$ (see, e.g., [2]) whose solutions $f: I \rightarrow \mathbb{R}$, for $I \subseteq \mathbb{R}$ containing the origin 0 , are of the form $f(x)=c x$, for some $c \in \mathbb{R}$, or the graph of $f$ is everywhere dense in $I \times \mathbb{R}$ (for a recent reference, see also [10]). Another particular instance of (3) is the so-called Jensen's equation $f\left(\frac{x+y}{2}\right)=\frac{f(x)+f(y)}{2}$ whose most general solutions are of the form $f(x)=f_{0}(x)+a$ for an arbitrary $a \in \mathbb{R}$ and an arbitrary function $f_{0}$ satisfying Cauchy's equation (see, e.g., [1]).

Example 4. Several other examples of functional equations, involving not only unary functions, can be found in classical theory of functional equations. A noteworthy example is the so-called Sincov's functional equation $f\left(x_{1}, x_{2}\right)+f\left(x_{2}, x_{3}\right)=$ $f\left(x_{1}, x_{3}\right)$. The general solutions for this $\left(\mathcal{L}_{0}, \mathcal{I}_{\mathbb{R}}\right)$-equation are known to be of the form $f\left(x_{1}, x_{2}\right)=g\left(x_{2}\right)-g\left(x_{1}\right)$. (Here $\mathcal{L}_{0}$ denotes the clone of 0 -preserving linear functions over the field $\mathbb{R}$.) For general background and further examples in classical theory of functional equations see, e.g., [1, 2, 17].

Example 5. For any $f \in B^{A^{n}}$, the singleton $\{f\}$ can be defined by $\left(\mathcal{C}_{B}, \mathcal{C}_{A}\right)$ equations as follows. Let a be an arbitrary element of $A^{n}$, and let $b=f(\mathbf{a})$. The functional equation $\mathbf{f}\left(a_{1}, \ldots, a_{n}\right)=b$ is a $\left(\mathcal{C}_{B}, \mathcal{C}_{A}\right)$-equation: on the left side the outer function is the identity function, and the inner functions are the constants $a_{1}, \ldots, a_{n}$, while on the right side we have only the constant function $b$ as outer function. These equations, for all $\mathbf{a} \in A^{n}$, constitute a system of equations that is satisfied only by $f$, since these equations specify the value of the function at every $\mathbf{a} \in A^{n}$.

Example 6. Let $A$ be a finite field, and let $\mathcal{L}_{0}$ be the clone of 0 -preserving linear functions on $A$, i.e., functions of the form $u\left(x_{1}, \ldots, x_{r}\right)=a_{1} x_{1}+\cdots+a_{r} x_{r}\left(a_{i} \in A\right)$. Let us examine which classes $\mathcal{K} \subseteq \mathcal{O}_{A}^{(n)}$ are definable by $\left(\mathcal{L}_{0}, \mathcal{C}_{A}\right)$-equations. Let 
$N=|A|^{n}$, and let $\left\{\mathbf{a}_{1}, \ldots, \mathbf{a}_{N}\right\}=A^{n}$. We will regard a function $f \in \mathcal{O}_{A}^{(n)}$ as a vector $\vec{f}$ in the $N$-dimensional vector space $A^{N}$ whose $i$-th coordinate is $f\left(\mathbf{a}_{i}\right)$. Any $\left(\mathcal{L}_{0}, \mathcal{C}_{A}\right)$-equation (for $\mathbf{f}=f$ ) translates into a system of homogeneous linear equations involving the components of $\vec{f}$ (namely, one equation corresponding to each assignment of values to the variables). Conversely, every homogeneous linear equation involving the components of $\vec{f}$ is equivalent to an $\left(\mathcal{L}_{0}, \mathcal{C}_{A}\right)$-equation. Therefore, a function class $\mathcal{K}$ is definable by $\left(\mathcal{L}_{0}, \mathcal{C}_{A}\right)$-equations if and only if the corresponding set $\overrightarrow{\mathcal{K}}=\{\vec{f}: f \in \mathcal{K}\}$ of vectors is the solution set of a system of homogeneous linear equations. It is a well-known fact from linear algebra that such sets can be characterized as subspaces of the vector space $A^{N}$. Thus, a class $\mathcal{K}$ of functions is definable by $\left(\mathcal{L}_{0}, \mathcal{C}_{A}\right)$-equations if and only if it is closed under linear combinations.

In the next section we make some general observations about equational definability, and in the last two sections we consider the problem of characterizing function classes definable by $(\mathcal{B}, \mathcal{A})$-equations by means of closure conditions, much in the spirit of Example 6. We present a necessary condition in Section 3, and we prove in Section 4 that this necessary condition is also sufficient in the four cases when $\mathcal{B}$ and $\mathcal{A}$ contain either only projections or all functions.

\section{Preliminary Results}

From the fact that $A$ and $B$ are finite it follows that definability by $(\mathcal{B}, \mathcal{A})$ equations can be always achieved by means of finite sets of $(\mathcal{B}, \mathcal{A})$-equations.

Proposition 1. A class $\mathcal{K} \subseteq B^{A^{n}}$ is definable by $(\mathcal{B}, \mathcal{A})$-equations if and only if it is finitely definable by $(\mathcal{B}, \mathcal{A})$-equations.

Proof. Clearly, the condition is sufficient. To show that it is also necessary, suppose that $\mathcal{K}$ is defined by a (possibly infinite) set $\mathcal{E}$ of $(\mathcal{B}, \mathcal{A}$ )-equations. For every $f \in B^{A^{n}} \backslash \mathcal{K}$ we can find an equation in $\mathcal{E}$ that is not satisfied by $f$. Choosing one such equation for each $f \in B^{A^{n}} \backslash \mathcal{K}$, we get a finite subset of $\mathcal{E}$ that defines $\mathcal{K}$, since $B^{A^{n}} \backslash \mathcal{K}$ is finite.

This result can be strengthened for sufficiently large clones $\mathcal{B}$. Indeed, as our next result indicates, if the clone $\mathcal{B}$ contains certain functions, then a single $(\mathcal{B}, \mathcal{A})$ equation suffices. To this extent, let 0 and 1 be two arbitrary distinct elements of $B$, and consider analogues of the Boolean conjunction $\wedge$ and equivalence $\leftrightarrow$, defined as follows: for $a, b \in A$ let

$$
\begin{aligned}
& a \wedge b= \begin{cases}1, & \text { if } a=1 \text { and } b=1 ; \\
0, & \text { otherwise; }\end{cases} \\
& a \leftrightarrow b= \begin{cases}1, & \text { if } a=b ; \\
0, & \text { otherwise. }\end{cases}
\end{aligned}
$$

Proposition 2. If clone $\mathcal{B}$ contains the operations $\wedge$ and $\leftrightarrow$, then a class $\mathcal{K} \subseteq B^{A^{n}}$ is definable by $(\mathcal{B}, \mathcal{A})$-equations if and only if it can be defined by a single $(\mathcal{B}, \mathcal{A})$ equation.

Proof. The sufficiency is obvious; for the necessity let us suppose that $\mathcal{K}$ is definable by $(\mathcal{B}, \mathcal{A})$-equations. By Proposition 1 , we may assume that $\mathcal{K}$ is defined by a finite set $\left\{E_{1}, \ldots, E_{t}\right\}$ of $(\mathcal{B}, \mathcal{A})$-equations, say, each $E_{i}$ being of the form $T_{i 1}=T_{i 2}$. Consider the $(\mathcal{B}, \mathcal{A})$-equation

$$
\bigwedge_{i \in[t]}\left(T_{i 1} \leftrightarrow T_{i 2}\right)=1
$$

(Note that since clone $\mathcal{B}$ contains $\leftrightarrow$, it also contains the operation $1=x_{1} \leftrightarrow x_{1}$ occurring on the right side of the above equation.) Obviously, this equation alone defines $\mathcal{K}$. 
The next proposition shows that the set of classes $\mathcal{K} \subseteq B^{A^{n}}$ definable by $(\mathcal{B}, \mathcal{A})$ equations constitutes a lattice under union and intersection, provided that the clone $\mathcal{B}$ is sufficiently large.

Proposition 3. If clone $\mathcal{B}$ contains the operations $\wedge$ and $\leftrightarrow$, and $\mathcal{K}, \mathcal{K}^{\prime} \subseteq B^{A^{n}}$ are definable by $(\mathcal{B}, \mathcal{A})$-equations, then so are $\mathcal{K} \cap \mathcal{K}^{\prime}$ and $\mathcal{K} \cup \mathcal{K}^{\prime}$.

Proof. Clearly, $\mathcal{K} \cap \mathcal{K}^{\prime}$ is definable by $(\mathcal{B}, \mathcal{A})$-equations, whenever both $\mathcal{K}$ and $\mathcal{K}^{\prime}$ are definable by $(\mathcal{B}, \mathcal{A})$-equations.

For the second claim, observe that by Proposition 2 there are two $(\mathcal{B}, \mathcal{A})$-equations $T_{1}=T_{2}$ and $T_{1}^{\prime}=T_{2}^{\prime}$ defining $\mathcal{K}$ and $\mathcal{K}^{\prime}$, respectively. Let us define a binary operation $\vee$ on $B$ by the formula $x \vee y=(x \wedge y) \leftrightarrow(x \leftrightarrow y)$. It is easy to see that the restriction of this operation to $\{0,1\}$ coincides with the Boolean disjunction operation, moreover $\vee \in \mathcal{B}$ by our assumption on $\mathcal{B}$. Consider the $(\mathcal{B}, \mathcal{A})$-equation

$$
\left(T_{1} \leftrightarrow T_{2}\right) \vee\left(T_{1}^{\prime} \leftrightarrow T_{2}^{\prime}\right)=1 .
$$

(Note that since clone $\mathcal{B}$ contains $\leftrightarrow$, it also contains the operation $1=x_{1} \leftrightarrow x_{1}$ occurring on the right side of the above equation.) It is straightforward to verify that a function $f$ satisfies this equation if and only if $f \in \mathcal{K} \cup \mathcal{K}^{\prime}$.

Remark 1. Let us note that in Propositions 2 and 3 , the operation $\wedge$ can be replaced by any binary operation whose restriction to $\{0,1\}$ coincides with the Boolean conjunction.

Proposition 4. A class $\mathcal{K} \subseteq B^{A^{n}}$ is definable by $\left(\mathcal{B}, \mathcal{O}_{A}\right)$-equations if and only if it is definable by $\left(\mathcal{B}, \mathcal{C}_{A}\right)$-equations.

Proof. Sufficiency is obvious. To show necessity, let us assume that $\mathcal{K}$ is defined by a set $\mathcal{E}$ of $\left(\mathcal{B}, \mathcal{O}_{A}\right)$-equations. For any $m$-ary equation $E \in \mathcal{E}$, let us evaluate $E$ at every tuple $\mathbf{a} \in A^{m}$. This way we get $|A|^{m}$ equalities, each of which can be regarded as a $\left(\mathcal{B}, \mathcal{C}_{A}\right)$-equation (the inner functions are the constants obtained by evaluating the inner functions of $E$ at $\mathbf{a}$ ). A function $f$ satisfies these equations if and only if it satisfies the original equation $E$. Unfolding each equation of $\mathcal{E}$ in this manner, we end up with a (large) set of $\left(\mathcal{B}, \mathcal{C}_{A}\right)$-equations that defines $\mathcal{K}$.

Remark 2. In equation (2), only the arity of $f$ is fixed; the arities of the inner and outer functions are arbitrary. However, by making use of the fact that the underlying set $A$ is finite, we can actually restrict these arities. To see this, let us consider a functional equation $E$ of the form $(2)$. Let us choose a map $\rho:[m] \rightarrow A$, and let us replace the variables $x_{i}$ in $E$ by new variables $y_{\rho(i)}$ for all $i \in[m]$. We denote the resulting $|A|$-ary equation by $E^{\rho}$. If a function $f$ satisfies $E$, then it also satisfies $E^{\rho}$, since every evaluation of $E^{\rho}$ is also an evaluation of $E$. Conversely, if $f$ satisfies $E^{\rho}$ for all $\rho \in A^{[m]}$, then it also satisfies $E$, since every evaluation of $E$ is an evaluation of some $E^{\rho}$. Thus every equation $E$ can be translated to a set of $|A|$-ary equations, i.e., we can always assume $m=|A|$ in (2). As an illustration, let $E$ be the ternary equation

$$
u\left(f\left(g_{1}\left(x_{1}, x_{2}, x_{3}\right)\right), f\left(g_{2}\left(x_{1}, x_{2}, x_{3}\right)\right)\right)=v\left(f\left(h\left(x_{1}, x_{2}, x_{3}\right)\right)\right)
$$

with $A=\{0,1\}$. The above procedure would translate $E$ to a set of 8 equations, but actually the three equations

$$
\begin{aligned}
& u\left(f\left(g_{1}\left(y_{0}, y_{0}, y_{1}\right)\right), f\left(g_{2}\left(y_{0}, y_{0}, y_{1}\right)\right)\right)=v\left(f\left(h\left(y_{0}, y_{0}, y_{1}\right)\right)\right), \\
& u\left(f\left(g_{1}\left(y_{1}, y_{0}, y_{0}\right)\right), f\left(g_{2}\left(y_{1}, y_{0}, y_{0}\right)\right)\right)=v\left(f\left(h\left(y_{1}, y_{0}, y_{0}\right)\right)\right), \\
& u\left(f\left(g_{1}\left(y_{0}, y_{1}, y_{0}\right)\right), f\left(g_{2}\left(y_{0}, y_{1}, y_{0}\right)\right)\right)=v\left(f\left(h\left(y_{0}, y_{1}, y_{0}\right)\right)\right)
\end{aligned}
$$

are sufficient, since for any $\mathbf{a} \in A^{3}$ at least one of $a_{1}=a_{2}, a_{2}=a_{3}$ or $a_{1}=a_{3}$ holds.

In a similar manner, we can also restrict the arities of the outer functions. Indeed, once we have fixed the arities of the inner functions to be $m=|A|$ as above, we have only $\left(|A|^{|A|^{m}}\right)^{n}$ possibilities for the $n$-tuples of inner functions $\left(g_{i 1}, \ldots, g_{i n}\right)$ 
appearing in $E$, and we can identify those variables of $u$ that carry the same $n$-tuple of inner functions. This way we can replace $u$ (and similarly $v$ ) by a function of arity at most $\left(|A|^{|A|^{m}}\right)^{n}=|A|^{n|A|^{|A|}}$, and by adding fictitious variables if necessary, we can actually assume that the arity of $u$ and $v$ is exactly $|A|^{n|A|^{|A|}}$. Observe that clones are closed under forming substitution instances of its members where variables are substituted for variables; hence the equations we obtain by performing the replacements of inner and outer functions as described above are still $(\mathcal{B}, \mathcal{A})$ equations. Thus, a class is definable by arbitrary $(\mathcal{B}, \mathcal{A})$-equations if and only if it is definable by $(\mathcal{B}, \mathcal{A})$-equations of the form $\sqrt{2}$ with $m=|A|$ and $r=s=|A|^{n|A|^{|A|}}$.

\section{A NECESSARY CONDITION FOR EQUATIONAL DEFINABILITY}

In this section we address the question: Which classes of functions are definable by $(\mathcal{B}, \mathcal{A})$-equations? In other words, we consider the following problem:

Problem 1. Given two clones $\mathcal{A}$ and $\mathcal{B}$, determine necessary and sufficient closure conditions on a class $\mathcal{K}$ which guarantee the existence of a defining set of $(\mathcal{B}, \mathcal{A})$ equations.

The general solution to this problem eludes us. However, we provide partial results towards a general solution of this problem. To this extent we need to recall some notions concerning certain special clones.

Two functions $f: A^{n} \rightarrow A$ and $g: A^{m} \rightarrow A$ are said to commute, denoted by $f \perp g$, if for all $a_{i j} \in A(i \in[n], j \in[m])$, we have

$$
\begin{aligned}
f\left(g\left(a_{11}, a_{12}, \ldots, a_{1 m}\right), \ldots,\right. & \left.g\left(a_{n 1}, a_{n 2}, \ldots, a_{n m}\right)\right) \\
= & g\left(f\left(a_{11}, a_{21}, \ldots, a_{n 1}\right), \ldots, f\left(a_{1 m}, a_{2 m}, \ldots, a_{n m}\right)\right) .
\end{aligned}
$$

The above definition of commutation is illustrated as follows: given any $n \times m$ matrix

$$
M=\left(\begin{array}{ccc}
a_{11} & \cdots & a_{1 m} \\
\vdots & & \vdots \\
a_{n 1} & \cdots & a_{n m}
\end{array}\right)
$$

over $A$, first applying $g$ to the rows of $M$ and then applying $f$ to the resulting column vector yields the same result as first applying $f$ to the columns of $M$ and then applying $g$ to the resulting row vector.

Let $\mathcal{A} \subseteq \mathcal{O}_{A}$ be a class of functions on $A$. The centralizer of $\mathcal{A}$, denoted by $\mathcal{A}^{*}$, is defined as the set of all functions which commute with every member of $\mathcal{A}$, i.e.,

$$
\mathcal{A}^{*}=\left\{g \in \mathcal{O}_{A}: g \perp f \text { for every } f \in \mathcal{A}\right\} .
$$

It is not difficult to verify that $\mathcal{A}^{*}$ is a clone for any class $\mathcal{A}$ (see, e.g., [16, 18, 25]). The clones $\mathcal{O}_{A}$ and $\mathcal{I}_{A}$ are centralizers of each other: $\mathcal{O}_{A}^{*}=\mathcal{I}_{A}$ and $\mathcal{I}_{A}^{*}=\mathcal{O}_{A}$.

The following proposition establishes necessary conditions for a class $\mathcal{K}$ to be definable by $(\mathcal{B}, \mathcal{A})$-equations.

Proposition 5. If a class $\mathcal{K} \subseteq B^{A^{n}}$ is definable by $(\mathcal{B}, \mathcal{A})$-equations, then

(A) for every $f \in \mathcal{K}$ and $\varphi \in\left(\mathcal{A}^{*}\right)^{(1)}$ we have $f\left(\varphi\left(x_{1}\right), \ldots, \varphi\left(x_{n}\right)\right) \in \mathcal{K}$, and

(B) for every $\ell \geq 0, f_{1}, \ldots, f_{\ell} \in \mathcal{K}$ and $\Phi \in\left(\mathcal{B}^{*}\right)^{(\ell)}$ we have $\Phi\left(f_{1}, \ldots, f_{\ell}\right) \in \mathcal{K}$.

Proof. Assume that $\mathcal{K}$ is definable by $(\mathcal{B}, \mathcal{A})$-equations, and let $E$ be one of the defining equations of $\mathcal{K}$, given in the form (2). For any function $f: A^{n} \rightarrow B$ and any tuple $\mathbf{a} \in A^{m}$, let $\operatorname{LHS}(f, \mathbf{a})$ and $\operatorname{RHS}(f, \mathbf{a})$ denote the left-hand side and the right-hand side of $E$, respectively, when evaluated for $\mathbf{f}=f$ at $\mathbf{a}$.

For the first claim, let $f \in \mathcal{K}, \varphi \in\left(\mathcal{A}^{*}\right)^{(1)}$, and let $f^{\prime}: A^{n} \rightarrow B$ be defined by

$$
f^{\prime}\left(x_{1}, \ldots, x_{n}\right)=f\left(\varphi\left(x_{1}\right), \ldots, \varphi\left(x_{n}\right)\right) .
$$


For $\mathbf{a} \in A^{m}$ let $\varphi(\mathbf{a}):=\left(\varphi\left(a_{1}\right), \ldots, \varphi\left(a_{m}\right)\right)$. Since $\varphi \perp g_{i j}$, it holds that $g_{i j}(\varphi(\mathbf{a}))=$ $\varphi\left(g_{i j}(\mathbf{a})\right)(i \in[r], j \in[n])$. Therefore, $\operatorname{LHS}(f, \varphi(\mathbf{a}))=\operatorname{LHS}\left(f^{\prime}, \mathbf{a}\right)$. A similar argument shows that $\operatorname{RHS}(f, \varphi(\mathbf{a}))=\operatorname{RHS}\left(f^{\prime}, \mathbf{a}\right)$. Since $f \in \mathcal{K}$, the equality $\operatorname{LHS}(f, \varphi(\mathbf{a}))=\operatorname{RHS}(f, \varphi(\mathbf{a}))$ holds for all $\mathbf{a} \in A^{m}$. From this it follows that $\operatorname{LHS}\left(f^{\prime}, \mathbf{a}\right)=\operatorname{RHS}\left(f^{\prime}, \mathbf{a}\right)$ for all $\mathbf{a} \in A^{m}$. This means that $f^{\prime}$ satisfies $E$, and we conclude that $f^{\prime} \in \mathcal{K}$.

For the second claim, let $\Phi \in\left(\mathcal{B}^{*}\right)^{(\ell)}, f_{1}, \ldots, f_{\ell} \in \mathcal{K}$. We need to prove that $f^{\prime}:=\Phi\left(f_{1}, \ldots, f_{\ell}\right)$ satisfies $E$, i.e., that $\operatorname{LHS}\left(f^{\prime}, \mathbf{a}\right)=\operatorname{RHS}\left(f^{\prime}, \mathbf{a}\right)$ for all $\mathbf{a} \in A^{m}$. Since $f_{1}, \ldots, f_{\ell} \in \mathcal{K}$, we have $\operatorname{LHS}\left(f_{i}, \mathbf{a}\right)=\operatorname{RHS}\left(f_{i}, \mathbf{a}\right)$ for $i=1, \ldots, \ell$; therefore

$$
\Phi\left(\operatorname{LHS}\left(f_{1}, \mathbf{a}\right), \ldots, \operatorname{LHS}\left(f_{\ell}, \mathbf{a}\right)\right)=\Phi\left(\operatorname{RHS}\left(f_{1}, \mathbf{a}\right), \ldots, \operatorname{RHS}\left(f_{\ell}, \mathbf{a}\right)\right)
$$

for all $\mathbf{a} \in A^{m}$. Let us consider the following $\ell \times r$ matrix over $B$ :

$$
\left(\begin{array}{ccc}
f_{1}\left(g_{11}, \ldots, g_{1 n}\right)(\mathbf{a}) & \ldots & f_{1}\left(g_{r 1}, \ldots, g_{r n}\right)(\mathbf{a}) \\
\vdots & & \vdots \\
f_{\ell}\left(g_{11}, \ldots, g_{1 n}\right)(\mathbf{a}) & \ldots & f_{\ell}\left(g_{r 1}, \ldots, g_{r n}\right)(\mathbf{a})
\end{array}\right) .
$$

Applying $\Phi$ to the columns and $u$ to the resulting row vector, we obtain $\operatorname{LHS}\left(f^{\prime}, \mathbf{a}\right)$, whereas applying $u$ to the rows and $\Phi$ to the resulting column vector, we obtain $\Phi\left(\operatorname{LHS}\left(f_{1}, \mathbf{a}\right), \ldots, \operatorname{LHS}\left(f_{\ell}, \mathbf{a}\right)\right)$. Since $\Phi \perp u$, we have

$$
\operatorname{LHS}\left(f^{\prime}, \mathbf{a}\right)=\Phi\left(\operatorname{LHS}\left(f_{1}, \mathbf{a}\right), \ldots, \operatorname{LHS}\left(f_{\ell}, \mathbf{a}\right)\right) .
$$

A similar argument shows that

$$
\operatorname{RHS}\left(f^{\prime}, \mathbf{a}\right)=\Phi\left(\operatorname{RHS}\left(f_{1}, \mathbf{a}\right), \ldots, \operatorname{RHS}\left(f_{\ell}, \mathbf{a}\right)\right) .
$$

From (4), (5), (6) it follows that $\operatorname{LHS}\left(f^{\prime}, \mathbf{a}\right)=\operatorname{RHS}\left(f^{\prime}, \mathbf{a}\right)$ for all $\mathbf{a} \in A^{m}$. Thus $f^{\prime}$ satisfies $E$.

Let us note that Example 6 illustrates that the above conditions are also sufficient when $B=A$ is a finite field, and $\mathcal{B}=\mathcal{L}_{0}, \mathcal{A}=\mathcal{C}_{A}$. Indeed, in this case $\mathcal{A}^{*}$ is the clone of idempotent functions on $A$, thus $\left(\mathcal{A}^{*}\right)^{(1)}$ contains only the identity function, hence $f\left(\varphi\left(x_{1}\right), \ldots, \varphi\left(x_{n}\right)\right)=f$ in condition $(\mathrm{A})$ of Proposition 5 , this condition holds trivially for any class $\mathcal{K}$. The $\ell$-ary elements of $\mathcal{B}^{*}$ are just the linear functionals on the vector space $A^{\ell}$, and these are all of the form $a_{1} x_{1}+$ $\cdots+a_{\ell} x_{\ell}\left(a_{i} \in A\right)$, hence $\mathcal{B}^{*}=\mathcal{B}=\mathcal{L}_{0}$. Therefore, condition (B) of the above proposition expresses the fact that $\mathcal{K}$ is closed under linear combinations. As we have seen in Example 6, this condition is necessary and sufficient for definability by $\left(\mathcal{L}_{0}, \mathcal{C}_{A}\right)$-equations.

The next example shows that the two necessary conditions given in Proposition 5 are not always sufficient.

Example 7. Let $B=A=\{0,1,2,3\}$, and let $v$ be the unary function on $A$ defined by $v(0)=v(1)=v(2)=0, v(3)=1$. Let $\mathcal{B}$ be the clone generated by $v$, let $\mathcal{A}$ be any clone on $A$, and let $\mathcal{K} \subseteq \mathcal{O}_{A}^{(0)}$ consist of the constants 0 and 1 . We claim that $\mathcal{K}$ satisfies the two conditions of Proposition 5. Indeed, Condition (A) is trivial, as $\mathcal{K}$ contains only constants. Condition (B) states that every function $\Phi \in \mathcal{B}^{*}$ preserves the set $\{0,1\} \subseteq B$. This is clear, since $\{0,1\}$ is exactly the range of $v$.

Now let us suppose that $\mathcal{K}$ is defined by a set $\mathcal{E}$ of $(\mathcal{B}, \mathcal{A})$-equations. Since $v(v(x))$ is constant 0 , every function $u \in \mathcal{B}$ is of the form $u(\mathbf{x})=x_{i}, u(\mathbf{x})=$ $v\left(x_{i}\right)$ or $u(\mathbf{x})=0$. As $\mathcal{K}$ contains only nullary functions, inner functions do not appear at all in our $(\mathcal{B}, \mathcal{A})$-equations, therefore, $\mathcal{E}$ consists of some of the equations $v(\mathbf{f})=\mathbf{f}, \mathbf{f}=0$ and $v(\mathbf{f})=0$. However, the equations $v(\mathbf{f})=\mathbf{f}$ and $\mathbf{f}=0$ both define the set $\{0\}$, while $v(\mathbf{f})=0$ defines the set $\{0,1,2\}$, hence no combination of these equations can define $\mathcal{K}=\{0,1\}$. This shows that $\mathcal{K}$ is not definable by $(\mathcal{B}, \mathcal{A})$-equations.

In order to construct an example where the inner functions play a crucial role, let us consider classes $\mathcal{K} \subseteq B^{A}$ of unary functions definable by $\left(\mathcal{I}_{B}, \mathcal{A}\right)$-equations, where $\mathcal{A}$ is the clone generated by a given permutation group $G \leq S_{A}$. Such an 
equation is of the form $\mathbf{f}(g(x))=\mathbf{f}(h(x))$ or $\mathbf{f}(g(x))=\mathbf{f}(h(y))$, where $g, h \in G$. Clearly, the latter equation is satisfied only by constant functions. The solutions of $\mathbf{f}(g(x))=\mathbf{f}(h(x))$ are described in the next lemma, for which we need to introduce the following notation. For any permutation $g \in S_{A}$, let $\pi_{g}$ be the equivalence relation on $A$ whose blocks are the cycles of $g$ (in other words: the orbits of the group generated by $g$ ).

Lemma 1. A function $f: A \rightarrow B$ satisfies the equation $\mathbf{f}(g(x))=\mathbf{f}(h(x))$ for given $g, h \in S_{A}$ if and only if

$$
\pi_{h g^{-1}} \subseteq \operatorname{ker} f
$$

Proof. Let us assume that $f$ satisfies $\mathbf{f}(g(x))=\mathbf{f}(h(x))$, and let $q=h g^{-1}$. Evaluating the equation at $x=g^{-1}(a)$ for an arbitrary $a \in A$, we obtain $f(a)=f(q(a))$. By iteration, we get $f(a)=f\left(q^{k}(a)\right)$ for any $a \in A$ and $k \in \mathbb{N}$. If $\left(a, a^{\prime}\right) \in \pi_{h g^{-1}}=$ $\pi_{q}$, then there exists $k \in \mathbb{N}$ such that $a^{\prime}=q^{k}(a)$, hence we have $f(a)=f\left(a^{\prime}\right)$, i.e., $\left(a, a^{\prime}\right) \in \operatorname{ker} f$.

Assume now that $\pi_{q} \subseteq \operatorname{ker} f$, and let $a \in A$. Since $q(g(a))=h(a)$, we have $(g(a), h(a)) \in \pi_{q}$, hence $(g(a), h(a)) \in \operatorname{ker} f$, and this means that $f(g(a))=$ $f(h(a))$ holds for all $a \in A$. Therefore $f$ satisfies the equation $\mathbf{f}(g(x))=\mathbf{f}(h(x))$.

If a class $\mathcal{K} \subseteq B^{A}$ is defined by a system of equations $\mathbf{f}\left(g_{i}(x)\right)=\mathbf{f}\left(h_{i}(x)\right)(i \in[t])$, then, according to the above lemma, the members of $\mathcal{K}$ are exactly those functions $f$ that satisfy each of the conditions $\pi_{h_{i} g_{i}^{-1}} \subseteq \operatorname{ker} f(i \in[t])$. These can be translated into a single condition $\bigvee_{i \in[t]} \pi_{h_{i} g_{i}^{-1}} \subseteq$ ker $f$, where $\vee$ denotes the join operation in the lattice of equivalence relations (transitive closure of the union). Also, taking into account equations of the form $\mathbf{f}(g(x))=\mathbf{f}(h(y))$, we obtain the following description of classes of unary functions definable by $\left(\mathcal{I}_{B}, \mathcal{A}\right)$-equations.

Proposition 6. Let $\mathcal{A}$ be the clone generated by a given permutation group $G \leq S_{A}$. Then a class $\mathcal{K} \subseteq B^{A}$ is definable by $\left(\mathcal{I}_{B}, \mathcal{A}\right)$-equations if and only if either $\mathcal{K}$ is the class of all constant functions or there exists a subset $Q \subseteq G$ such that

$$
\mathcal{K}=\left\{f \in B^{A}: \bigvee_{q \in Q} \pi_{q} \subseteq \operatorname{ker} f\right\} .
$$

With the help of Proposition 6, we can give another example showing that the conditions of Proposition 5 do not always guarantee definability by $(\mathcal{B}, \mathcal{A})$ equations. In contrast to Example 7, here the key role is played by the inner functions.

Example 8. Let $\mathcal{A}$ be the clone generated by the alternating group on $A=$ $\{0,1,2,3\}$, and let $\mathcal{B}=\mathcal{I}_{B}$, where $B$ is an arbitrary set with at least two elements. Then the class $\mathcal{K}=\left\{f \in B^{A}: f(0)=f(1)\right\}$ satisfies the two conditions of Proposition 5, but it is not definable by $(\mathcal{B}, \mathcal{A})$-equations. To see this, let us observe that $\mathcal{K}=\left\{f \in B^{A}: \vartheta \subseteq \operatorname{ker} f\right\}$, where $\vartheta$ denotes the equivalence relation on $A$ whose blocks are $\{0,1\},\{2\},\{3\}$. From Proposition 6 it follows that $\mathcal{K}$ is definable by $(\mathcal{B}, \mathcal{A})$-equations if and only if $\vartheta$ is the join of some equivalences $\pi_{q}$ with each $q$ being an even permutation. However, $\vartheta$ is an atom in the lattice of equivalence relations on $A$, hence it is join irreducible. Therefore, if $\mathcal{K}$ was definable by $(\mathcal{B}, \mathcal{A})$-equations, then $\vartheta$ would be equal to $\pi_{q}$ for some even permutation $q$. This is clearly not the case, as the only permutation $q$ with $\pi_{q}=\vartheta$ is the transposition (01), which is an odd permutation. This shows that $\mathcal{K}$ is not definable by $(\mathcal{B}, \mathcal{A})$-equations.

To verify that $\mathcal{K}$ satisfies the conditions of Proposition 5 , let us observe that $\left(\mathcal{A}^{*}\right)^{(1)}$ contains only the identity function 1 , hence condition (A) is trivially satisfied.

\footnotetext{
${ }^{1}$ It is straightforward to check that the only unary function commuting with all even permutations is the identity, whenever the underlying set has at least 4 elements. Alternatively, one can use the description of the centralizers of alternating groups given by Machida and Rosenberg [21.
} 
As $\mathcal{I}_{B}^{*}=\mathcal{O}_{B}$, condition (B) is equivalent to condition (7) below, and we will see in Remark 4 that it is satisfied if and only if $\mathcal{K}$ is of the form $\mathcal{K}=\left\{f \in B^{A}: \vartheta \subseteq \operatorname{ker} f\right\}$ for some equivalence relation $\vartheta$, which is indeed the case in our example.

Remark 3. In light of Remark 2 the conditions of Proposition 5 can be slightly strengthened. Namely, condition (A) must hold for every unary function $\varphi$ that commutes with all $|A|$-ary members of $\mathcal{A}$, and similarly, in condition (B) it suffices to require that $\Phi$ commutes with the $|A|^{n|A|^{|A|}}$-ary part of $\mathcal{B}$. However, as Examples 7 and 8 show, these stronger necessary conditions are still not sufficient for definability by $(\mathcal{B}, \mathcal{A})$-equations.

\section{Characterizations of Classes Definable By EQuations induced By THE SMALLEST AND LARGEST CLONES}

In the rest of the paper we show that the necessary conditions presented in Proposition 5 are sufficient when $\mathcal{B} \in\left\{\mathcal{I}_{B}, \mathcal{O}_{B}\right\}$ and $\mathcal{A} \in\left\{\mathcal{I}_{A}, \mathcal{O}_{A}\right\}$. As $\mathcal{O}_{B}^{*}=\mathcal{I}_{B}$, in the case $\mathcal{B}=\mathcal{O}_{B}$ condition (B) is satisfied by every class, while for $\mathcal{B}=\mathcal{I}_{B}$ it takes the form

$$
\forall f_{1}, \ldots, f_{\ell} \in \mathcal{K} \forall \Phi \in \mathcal{O}_{B}^{(\ell)}: \Phi\left(f_{1}, \ldots, f_{\ell}\right) \in \mathcal{K},
$$

since $\mathcal{I}_{B}^{*}=\mathcal{O}_{B}$. Similarly, condition (A) is trivial for $\mathcal{A}=\mathcal{O}_{A}$, and for $\mathcal{A}=\mathcal{I}_{A}$ it reads as

$$
\forall f \in \mathcal{K} \forall \varphi \in \mathcal{O}_{A}^{(1)}: f\left(\varphi\left(x_{1}\right), \ldots, \varphi\left(x_{n}\right)\right) \in \mathcal{K} .
$$

Lemma 2. A class $\mathcal{K} \subseteq B^{A^{n}}$ is definable by $\left(\mathcal{B}, \mathcal{I}_{A}\right)$-equations if and only if it is definable by $\left(\mathcal{B}, \mathcal{O}_{A}\right)$-equations and satisfies condition (8).

Proof. The necessity of the conditions follows from Proposition 5 . To prove the sufficiency, let us suppose that $\mathcal{K} \subseteq B^{A^{n}}$ is definable by $\left(\mathcal{B}, \mathcal{O}_{A}\right)$-equations and satisfies (8). By Proposition 4, there exists a system $\mathcal{E}$ of $\left(\mathcal{B}, \mathcal{C}_{A}\right)$-equations that defines $\mathcal{K}$, moreover, we may assume that only constant functions appear as inner functions in every equation of $\mathcal{E}$. To simplify notation, in the following we will assume that $A=\{1,2, \ldots, k\}$. Let us replace every occurrence of every constant $i \in A$ by the $i$-th $k$-ary projection $x_{i}^{(k)}$ in every equation of $\mathcal{E}$. This way we obtain a system $\mathcal{E}^{\prime}$ of $k$-ary $\left(\mathcal{B}, \mathcal{I}_{A}\right)$-equations. We will prove that $\mathcal{E}^{\prime}$ defines $\mathcal{K}$.

Assume first that a function $f$ satisfies $\mathcal{E}^{\prime}$. Then the two sides of each equation of $\mathcal{E}^{\prime}$ evaluate to the same value for $\mathbf{f}=f$ at every $\mathbf{a} \in A^{k}$. Choosing $\mathbf{a}=(1,2, \ldots, k)$, we get exactly the original equations in $\mathcal{E}$. Therefore $f$ satisfies $\mathcal{E}$, and this implies that $f \in \mathcal{K}$.

Now let us assume that $f \in \mathcal{K}$, and let us evaluate the two sides of an equation in $\mathcal{E}^{\prime}$ for $\mathbf{f}=f$ at an arbitrary tuple $\mathbf{a} \in A^{k}$. Then we obtain the same values as if we evaluated the two sides of the corresponding equation of $\mathcal{E}$ for the function $\mathbf{f}=f\left(\varphi\left(x_{1}\right), \ldots, \varphi\left(x_{n}\right)\right)$, where $\varphi$ is the unary map defined by $\varphi(i)=a_{i}$ for every $i \in A$. Since $\mathcal{K}$ has property (8), $f\left(\varphi\left(x_{1}\right), \ldots, \varphi\left(x_{n}\right)\right) \in \mathcal{K}$, hence it satisfies every equation of $\mathcal{E}$. This shows that $f$ satisfies every equation of $\mathcal{E}^{\prime}$.

Theorem 1. Every class $\mathcal{K} \subseteq B^{A^{n}}$ is definable by $\left(\mathcal{O}_{B}, \mathcal{O}_{A}\right)$-equations.

Proof. We have seen in Example 5 that $\{f\}$ is definable for any given $f \in B^{A^{n}}$. Since $A$ and $B$ are finite sets, any class $\mathcal{K} \subseteq B^{A^{n}}$ is a finite union of singletons, hence the theorem follows by Proposition 3 .

Theorem 2. A class $\mathcal{K} \subseteq B^{A^{n}}$ is definable by $\left(\mathcal{O}_{B}, \mathcal{I}_{A}\right)$-equations if and only if it satisfies condition (8).

Proof. Follows from Lemma 2 and Theorem 1.

Theorem 3. A class $\mathcal{K} \subseteq B^{A^{n}}$ is definable by $\left(\mathcal{I}_{B}, \mathcal{O}_{A}\right)$-equations if and only if it satisfies condition (7). 
Proof. The necessity of the condition follows from Proposition 5. For the sufficiency, let us assume that $\mathcal{K} \subseteq B^{A^{n}}$ satisfies $(7)$, and let $f \in B^{A^{n}}$ be an arbitrary function that satisfies every $\left(\mathcal{I}_{B}, \mathcal{O}_{A}\right)$-equation that is satisfied by all members of $\mathcal{K}$. We will prove that $f \in \mathcal{K}$.

Let us choose $\ell=|\mathcal{K}|$, let $\mathcal{K}=\left\{f_{1}, \ldots, f_{\ell}\right\}$, and for any $\mathbf{a} \in A^{n}$ let us write $F(\mathbf{a})$ for the $\ell$-tuple $\left(f_{1}(\mathbf{a}), \ldots, f_{\ell}(\mathbf{a})\right)$. Let 0 be an arbitrary element of $A$, and for any $\mathbf{b} \in B^{\ell}$ let

$$
\Phi(\mathbf{b})= \begin{cases}f(\mathbf{a}), & \text { if } \mathbf{b}=F(\mathbf{a}) \\ 0, & \text { if } \nexists \mathbf{a} \in A^{n}: \mathbf{b}=F(\mathbf{a}) .\end{cases}
$$

We claim that this formula gives rise to a well-defined function $\Phi \in \mathcal{O}_{B}^{(\ell)}$. Suppose that $\mathbf{b}=F(\mathbf{a})=F\left(\mathbf{a}^{\prime}\right)$ for some tuples $\mathbf{a}, \mathbf{a}^{\prime} \in A^{n}$. Let us consider the functional equation

$$
\mathbf{f}\left(a_{1}, \ldots, a_{n}\right)=\mathbf{f}\left(a_{1}^{\prime}, \ldots, a_{n}^{\prime}\right) .
$$

This is an $\left(\mathcal{I}_{B}, \mathcal{O}_{A}\right)$-equation, since the outer function on both sides is the identity function (i.e., the first unary projection), and the inner functions are constants. The equality $F(\mathbf{a})=F\left(\mathbf{a}^{\prime}\right)$ implies that every element of $\mathcal{K}$ satisfies $(9)$. Therefore, according to our assumption, $f$ satisfies $(9)$ as well. This means that $f(\mathbf{a})=f\left(\mathbf{a}^{\prime}\right)$, hence $\Phi(\mathbf{b})=f(\mathbf{a})=f\left(\mathbf{a}^{\prime}\right)$ is well defined.

Since $\mathcal{K}$ satisfies condition $(7)$, in order to prove that $f \in \mathcal{K}$, it suffices to verify that $f=\Phi\left(f_{1}, \ldots, f_{\ell}\right)$. Indeed, for all $\mathbf{a} \in A^{n}$ we have

$$
\Phi\left(f_{1}, \ldots, f_{\ell}\right)(\mathbf{a})=\Phi(F(\mathbf{a}))=f(\mathbf{a})
$$

by the definition of $\Phi$.

Remark 4. As the proof of Theorem 3 shows, a class $\mathcal{K} \subseteq B^{A^{n}}$ satisfies condition (7) if and only if it can be defined by equations of the form (9), i.e., by equations that involve only constants as inner functions (cf. also Proposition 4). A function $f$ satisfies equation (9) if and only if $f(\mathbf{a})=f\left(\mathbf{a}^{\prime}\right)$, which is equivalent to $\left(\mathbf{a}, \mathbf{a}^{\prime}\right) \in$ ker $f$. Therefore, the assertion that a given set $\mathcal{E}$ of equations of this form is satisfied by a function $f$ is equivalent to the condition $R \subseteq \operatorname{ker} f$, where $R$ is a binary relation on $A^{n}$ (consisting of all pairs $\left(\mathbf{a}, \mathbf{a}^{\prime}\right)$ that appear in $\mathcal{E}$ ). If $\vartheta$ is the least equivalence relation containing $R$, then clearly $R \subseteq \operatorname{ker} f$ if and only if $\vartheta \subseteq \operatorname{ker} f$. Thus a class $\mathcal{K} \subseteq B^{A^{n}}$ satisfies $(7)$ if and only if there exists an equivalence relation $\vartheta$ on $A^{n}$ such that

$$
\mathcal{K}=\left\{f \in B^{A^{n}}: \vartheta \subseteq \operatorname{ker} f\right\} .
$$

Theorem 4. A class $\mathcal{K} \subseteq B^{A^{n}}$ is definable by $\left(\mathcal{I}_{B}, \mathcal{I}_{A}\right)$-equations if and only if conditions (7) and (8) hold.

Proof. Follows from Lemma 2 and Theorem 3

\section{ACKNOWLEDGMENT}

The authors would like to thank Eszter K. Horváth for helpful discussions.

The first named author is supported by the internal research project F1R-MTHPUL-09MRDO of the University of Luxembourg. The third named author acknowledges that the present project is supported by the TÁMOP-4.2.1/B-09/1/KONV2010-0005 program of National Development Agency of Hungary, by the Hungarian National Foundation for Scientific Research under grants no. K77409 and K83219, by the National Research Fund of Luxembourg, and cofunded under the Marie Curie Actions of the European Commission (FP7-COFUND).

\section{REFERENCES}

[1] J. Aczél, Lectures on Functional Equations and Their Applications, Dover Publications Inc., New York, 1986.

[2] J. Aczél and J. Dhombres, Functional Equations in Several Variables, Encyclopedia of Mathematics and Its Applications, vol. 31, Cambridge University Press, Cambridge, 1989. 
[3] V. G. Bodnarčuk, L. A. Kalužnin, V. N. Kotov and B. A. Romov, Galois theory for Post algebras. I, II Kibernetika 3 (1969) 1-10, 5 (1969) 1-9 (in Russian). English translation: Cybernetics 5 (1969) 243-252, 531-539.

[4] M. Couceiro, On the lattice of equational classes of Boolean functions and its closed intervals, J. Mult.-Valued Logic Soft Comput. 18 (2008) 81-104.

[5] M. Couceiro and S. Foldes, Definability of Boolean function classes by linear equations over GF(2), Discrete Appl. Math. 142 (2004) 29-34.

[6] M. Couceiro and S. Foldes, On closed sets of relational constraints and classes of functions closed under variable substitutions, Algebra Universalis 54 (2005) 149-165.

[7] M. Couceiro and S. Foldes, Functional equations, constraints, definability of function classes, and functions of Boolean variables, Acta Cybernet. 18 (2007) 61-75.

[8] M. Couceiro and S. Foldes, Function classes and relational constraints stable under compositions with clones, Discuss. Math. Gen. Algebra Appl. 29 (2009) 109-121.

[9] M. Couceiro, E. Lehtonen, and T. Waldhauser, On equational definability of function classes, 41st IEEE International Symposium on Multiple-Valued Logic (ISMVL 2011), IEEE Computer Society, 2011, pp. 182-186.

[10] M. Couceiro and J.-L. Marichal, Axiomatizations of Lovász extensions of pseudo-Boolean functions, Fuzzy Sets and Systems 181 (2011) 28-38.

[11] M. Couceiro and M. Pouzet, On a quasi-ordering on Boolean functions, Theoret. Comput. Sci. 396 (2008) 71-87.

[12] O. Ekin, S. Foldes, P. Hammer, and L. Hellerstein, Equational characterizations of Boolean function classes, Discrete Math. 211 (2000) 27-51.

[13] S. Foldes, Equational classes of Boolean functions via the HSP theorem, Algebra Universalis 44 (2000) 309-324.

[14] S. Foldes and G. R. Pogosyan, Post classes characterized by functional terms, Discrete Appl. Math. 142 (2004) 35-51.

[15] D. Geiger, Closed systems of functions and predicates, Pacific J. Math. 27 (1968) 95-100.

[16] M. Hermann, On Boolean primitive positive clones, Discrete Math. 308 (2008) 3151-3162.

[17] M. Kuczma, An Introduction to the Theory of Functional Equations and Inequalities. Cauchy's Equation and Jensen's Inequality, (2nd ed.) Birkhäuser Verlag, Basel, 2009.

[18] A. V. Kuznecov, On detecting non-deducibility and non-expressibility, Logical deduction, Nauka, Moscow, 1979, pp. 5-33 (in Russian).

[19] D. Lau, Function Algebras on Finite Sets, Springer Monographs in Mathematics, SpringerVerlag, Berlin, 2006.

[20] L. Lovász, Submodular functions and convexity, Mathematical Programming: The state of the art (Bonn, 1982), Springer, Berlin, 1983, pp. 235-257.

[21] H. Machida and I. G. Rosenberg, On the centralizers of monoids in clone theory, 33rd IEEE International Symposium on Multiple-Valued Logic (ISMVL 2003), IEEE Computer Society, 2003, pp. 303-308.

[22] S. S. Marchenkov and V. S. Fedorova, Solutions of systems of functional equations of manyvalued logic, Moscow Univ. Comput. Math. Cybernet. 33 (2009) 197-201.

[23] N. Pippenger, Galois theory for minors of finite functions, Discrete Math. 254 (2002) 405419.

[24] R. Pöschel and L. A. Kalužnin, Funktionen- und Relationenalgebren, Mathematische Monographien, VEB Deutscher Verlag der Wissenschaften, Berlin, 1979.

[25] L. Szabó, On the lattice of clones acting bicentrally, Acta Cybernet. 6 (1984) 381-388.

[26] D. M. Topkis, Minimizing a submodular function on a lattice, Operations Res. 26 (1978) 305-321.

[27] D. J. A. Welsh, Matroid Theory, L. M. S. Monographs, No. 8., Academic Press, London, New York, 1976.

(M. Couceiro) Mathematics Research Unit, University of Luxembourg, 6, Rue Richard Coudenhove-Kalergi, L-1359 Luxembourg, Luxembourg and LAMSADE - CNRS, Université Paris-Dauphine, Place du Maréchal de lattre de Tassigny, 75775 Paris Cedex 16, FRANCE

E-mail address: miguel.couceiro@dauphine.fr

(E. Lehtonen) Computer Science and Communications Research Unit, University of Luxembourg, 6, Rue Richard Coudenhove-Kalergi, L-1359 Luxembourg, Luxembourg and Centro de Álgebra da Universidade de Lisboa, Avenida Professor Gama Pinto 2, 1649 003 Lisboa, Portugal

E-mail address: erkko@campus.ul.pt

(T. Waldhauser) Mathematics Research Unit, University of Luxembourg, 6, RUe Richard Coudenhove-Kalergi, L-1359 Luxembourg, Luxembourg and Bolyai Institute, University of Szeged, Aradi vértanúk tere 1, H-6720 Szeged, Hungary

E-mail address: twaldha@math.u-szeged.hu 